\title{
Effect of perioperative cognitive behavioral therapy on chronic post-surgical pain among breast cancer patients with high pain catastrophising characteristics: Protocol for a double-blinded randomised controlled trial.
}

Aneurin Moorthy ( $\square$ aneurin.moorthy@gmail.com )

Mater University Hospital

\section{Damien Lowry}

Mater Misericordiae University Hospital

\section{Carla Edgley}

University College Dublin - National University of Ireland: University College Dublin

\section{Máire-Bríd Casey}

Mater Misericordiae University Hospital

\section{Donal J. Buggy}

Mater Misericordiae University Hospital

\section{Study protocol}

Keywords: Breast cancer surgery, chronic postsurgical pain, mastectomy, cognitive behavioural therapy, psychological factors, pain catastrophizing, anxiety

Posted Date: September 10th, 2021

DOl: https://doi.org/10.21203/rs.3.rs-892586/v1

License: @ (i) This work is licensed under a Creative Commons Attribution 4.0 International License. Read Full License 


\section{Abstract}

Background

Surgery is regarded as the primary treatment for breast cancer. Chronic post-surgical pain (CPSP) is a recognised complication after breast cancer surgery, and it is estimated to affect $20-30 \%$ of women. Pain catastrophizing has emerged as one of the most influential psychological variables associated with CPSP.

Methods

This trial will be a single-centre, prospective, double-blinded, noninferiority, randomised controlled trial (RCT). Patients scheduled for elective breast cancer surgery (wide local excision or mastectomy with or without axillary lymph node dissection) will be screened preoperatively for high pain catastrophising. Patients with high pain catastrophising, defined as a score of $\geq 24$ on the Pain Catastrophising Scale will be deemed eligible for inclusion in the study. Participants will be randomly assigned to receive either a cognitive behavioural therapy or an educational mindfulness based program during their perioperative period. The primary outcome is the Brief Pain Inventory short form average pain severity score at three months postoperatively. Secondary outcomes include patient-reported quality of recovery at Day 1-2 after surgery, levels of pain catastrophising, reported depressed mood and anxiety. ]

Discussion

To the best of our knowledge, this protocol describes the first RCT which directly examines the effect of perioperative cognitive behavioural therapy on CPSP among breast cancer patients with high pain catastrophising characteristics. The outcomes of this trial may have significant implications for these patients because perioperative cognitive behavioural therapy has the potential to become an important perioperative intervention to complement patient management.

Trial registration

This trial is registered on ClinicalTrials.gov Identifier: NCT04924010 on 11th June 2021. All item from the World Health Organisation Trial Registration Data set have been included.

https://clinicaltrials.gov/ct2/show/NCT04924010

\section{Introduction}

\section{Background and rationale $\{6 a\}$}

Chronic post-surgical pain (CPSP) is defined as pain at or near the site of surgery persisting for 3 months or more after the date of surgery. The incidence of CPSP in Europe is up to $50 \%$ at 3 months and $12 \%$ at 12 months, but the incidence varies depending on surgical procedure(1). In Breast surgery, one of the most commonly performed surgical procedures for cancer(2), CPSP has been observed in 20-30\% of patients at 6 months after surgery, making this group among the highest risk of developing $\operatorname{CPSP}(3,4)$. Clinical developments that could mitigate the development of CPSP following breast cancer surgery would potentially 
yield multiple benefits in terms of reducing future healthcare utilisation, associated costs ${ }^{3}$, and improving physical and mental health outcomes in this patient cohort.

Several predictive factors for CPSP have been identified, the most important being the presence of chronic pre-operative pain, high intensity of acute postoperative pain and several psychological factors(5). Of these psychological factors, pain catastrophizing has emerged as one of the strongest predictors of pain severity and disability among individuals with a range of pain presentations and CPSP(6-9). Pain catastrophizing is described as a maladaptive psychological coping strategy involving an exaggerated reaction to anticipated or actual pain. It can involve mental rumination, magnification of the perceived danger or threat associated with pain, and feelings of helplessness in relation to what can be done $(6,9)$. A recent systematic review on psychological interventions in patients undergoing major elective abdominal surgery concluded that pain catastrophising can have a direct influence on the neuropathophysiological mechanisms underlying pain experiences and can improve pain and psychological outcomes, after surgery(10).

Psychological variables are modifiable, and catastrophizing appears to be an exciting clinical target for intervention. In recent years, there has been a growing number of studies investigating the potential impact of perioperative psychological interventions in a variety of patient groups. A recent systematic review and metaanalysis of observational studies concluded that psychological predictors may have a significant association with chronic post-surgical pain, including catastrophization, although this conclusion is limited by the heterogeneity of study designs, methods used and a lack of robust randomised controlled trial (RCT) data to help delineate causative links(11).

\section{Objectives $\{7\}$}

The primary objective of this RCT is to examine whether a perioperative Cognitive Behavioural Therapy (CBT) based psychological intervention is effective at reducing chronic pain intensity at three-month follow up, in high catastrophising patients undergoing breast cancer surgery, as compared to a pain education and mindfulness programme.

Secondary objectives of the study include examining whether perioperative CBT has an impact in reducing pain interference, quality-of-recovery, pain catastrophising, depression and anxiety compared to a pain education and mindfulness programme.

\section{Trial design \{8\}}

This is a single-centre, prospective, double-blinded, noninferiority, randomised controlled trial (RCT).

The study will randomise breast cancer surgery patients into two groups. One group will receive four sessions of perioperative cognitive behavioural therapy (CBT) in addition to usual care, and the other group will receive four perioperative sessions of pain education and mindfulness exercises. Figure 1 illustrates the study flow.

\section{Methods: Participants, Interventions, And Outcomes}

\section{Study setting $\{9\}$}


This study will be conducted in a tertiary university hospital in Dublin (Mater Misericordiae University Hospital), Republic of Ireland. The hospital performs over 500 breast cancer surgeries per year.

\section{Eligibility criteria $\{10\}$}

Inclusion criteria are:

- Female patients

- Aged 18-75

- Undergoing breast cancer surgery (either wide local excision with magseed or full mastectomy)

- Pain Catastrophising Scale (PCS) score of 24 or higher

Exclusion criteria are:

- Pain Catastrophising Scale score below 24

- Surgery for benign breast disease

- Patient non-consent

- Plans to undergo major surgery within six months after current breast surgery

- Comorbid severe psychiatric conditions such as schizophrenia or personality disorder

- Known or suspected non-compliance

- Known or suspected drug or alcohol abuse problems within past 3 months

- Inability to follow the study procedures e.g. dementia or non-fluency of English

- The presence of any serious medical comorbidity that might cause disability or worsen the patient's general health condition

- Pregnancy

- An opioid intrathecal pump

- Cognitive behavioural therapy in the past 12 months.

- Inability to complete at least one psychological session prior to breast cancer surgery

\section{Who will take informed consent? $\{26 \mathrm{a}\}$}

The senior pain psychologist (DL) delivering the perioperative interventions for this trial will screen for potential trial participants, after breast cancer surgery clinic nurses identify potential candidates for the study. Patients that meet the eligibility criteria will be contacted, and informed consent will be obtained. All potential participants will have the opportunity to withdraw at any time point during the study period.

\section{Additional consent provisions for collection and use of participant data and biological specimens $\{26 \mathrm{~b}\}$}

Not applicable. No data and biological specimens will be collected for use in ancillary studies 
CBT is a form of psychological treatment that is widely used to treat various mental health disorders. We aim

to examine its effectiveness in reducing pain intensity and development of chronic post-surgical pain (CPSP) three months post-breast cancer surgery. To achieve this, we have designed our trial to compare CBT with an active control arm, a pain education and mindfulness programme. By taking this approach, the study is controlled for the following factors: time exposure to the psychologist, pain education content and mindfulness practice. This will help to specifically isolate the potential effects of CBT on those participants in that arm of the study.

\section{Intervention description \{11a\}}

\section{Group 1: Cognitive Behavioural Therapy for chronic pain (CBT-CP)}

The CBT intervention will be delivered by a Senior Psychologist with seventeen years of hospital psychology experience including seven in the treatment of chronic pain patients. Sessions will be delivered in one-hour, individual therapy appointments to patients, and there will be an emphasis on mindfulness based stress reduction, cognitive restructuring, exercise and pacing, behavioural activation, improving sleep, and anger management. Standardized worksheets and homework assignments are an important part of CBT-CP, and these will be given to patients who will be asked to read and complete in terms of complementing the consultations. A total of four sessions will be delivered during the perioperative period, with at least one of these taking place prior to surgery. Further details of each individual session and its content are provided in Table 1. This intervention has been developed with reference to two sources: A successful national implementation of CBT-CP using video teleconferencing format (12) and an evidence-based CBT manual specifically designed to treat chronic pain (13).

\section{Group 2: Pain Education and Mindfulness (PEM)}

To control for the potential effects of additional time spent with a professional, pain education and mindfulness input between the two interventions, patients in the education and mindfulness group will also have four perioperative psychology sessions, at least one of which will take place before the surgery. As the same person will be providing the CBT-CP and educational interventions, this potential confounding factor will also be controlled for. The control intervention consists of discussing pain education, as derived from the selfmanagement section of the chronic pain Ireland website (https://www.chronicpain.ie/our-services/selfmanagement) and the persistent pain section of the pain toolkit website (https://www.paintoolkit.org/persistent-pain). Four mindfulness-based stress reduction exercises will be completed including an introductory session on mindful breathing, a guided meditation, progressive muscle relaxation and a body scan. These sessions will each last less than one hour.

\section{Criteria for discontinuing or modifying allocated interventions $\{11 \mathrm{~b}\}$}

Criteria for discontinuing the allocated interventions are:

1. Patient withdrawal of consent at any point of study

2. Worsening mental health or psychological well-being of patient 


\section{Strategies to improve adherence to interventions $\{11 \mathrm{c}\}$}

All participants for this trial will receive a total of four psychological based interventions (see 'interventions description' part 11a). Each intervention session lasts for approximately 60 minutes. To ensure patient adherence to the study interventions during the trial period, all participants will be well-informed during the consent process. The study participation burden, such as duration and timing of each psychology session, and completion of primary outcome questionnaires at one and, three months post-surgery will be explained. The senior pain psychologist responsible for screening potential study participants will record all eligible candidates, and those that don't proceed with the study due to ineligibility, non-consent, or withdrawal. In so far as it is possible, only eligible, and fully willing individuals will proceed to randomisation and allocation of the patient to either the CBT-CP or PEM group.

\section{Relevant concomitant care permitted or prohibited during the trial $\{11 \mathrm{~d}\}$}

All patients will receive standard peri-operative surgical and anaesthesia care during this trial. These include:

1. Pre-operative assessment: This may involve optimization of underlying medical conditions (e.g., Blood pressure or glucose control)

2. Intra-operative interventions: All patients will receive a general anaesthesia as part of their surgical management. This may be complemented with regional anaesthesia techniques (e.g., PEC Block) for post-operative pain management, this will be under the discretion of the treating anaesthesiologist.

3. Post-operative interventions: All patient will have a post-operative analgesia plan. Again, this will be under the discretion of the treating anaesthesiologists. Standard post-operative analgesia that may be prescribed but are not limited to include paracetamol, Non-steroidal anti-inflammatory drug, morphine, fentanyl and gabapentin.

\section{Provisions for post-trial care $\{30\}$}

All participants that are enrolled into this study are covered by indemnity for negligent harm, through the standard Health Service Executive (HSE) indemnity arrangements. If any participant suffers from any stress or mental health complications arising directly from either intervention during or after the trial, then the participant will be offered further psychological management in line with standard care. This will be offered by a different clinical psychologist, that has no direct or indirect involvement with this trial. The research team for this study will liaise with clinical staff attached to the breast cancer surgery teams in order to make these arrangements.

\section{Outcomes $\{12\}$}

\section{Primary outcome:}

1. Brief Pain Inventory Short form: average pain severity score. [ Time Frame: 3 months postoperative].

- Brief Pain Inventory (BPI) Short form will be used to assess for CPSP after Breast Cancer Surgery. This assessment will be conducted by a member of the research team. BPI assess for quality of life and pain and its scale is measured between 0 - 10 , where ' 0 ' indicates no pain and ' 10 ' indicates severe pain. A 
decrease in the BPI score of 2 or more from the baseline score is considered clinically significant and indicates an improvement in severity of the patient's cancer and or non-cancer pain (14)

\section{Secondary outcomes:}

Secondary outcomes of the study include pain interference, quality of recovery from surgery, levels of pain catastrophising, reported depressed mood and levels of anxiety. These will be measured as follows:

- Brief Pain Inventory Short form: average pain interference score. [ Time Frame: 3 months postoperative].

i. BPI average pain interference score assess for interference the pain has on the patient's functioning. BPI is measured between $0-10$, where ' 0 ' indicates no interference and ' 10 ' indicates severe interference with quality of life (14).

- Quality of Recovery-15 (QoR-15)[Time Frame: 24-48 hours, post-operative]

- QoR-15 is a 15-item questionnaire which is used as a tool to assess overall patient recovery and pain after surgery. Participant will be asked to complete this questionnaire within 24-48 hours after their surgery. It is scored between 0 and 150, where the greater the number indicates excellent postoperative recovery (15).

- Pain Catastrophising Scale (PCS).[Time Frame: 1 and 3 months, post-operative]

- PCS is a 13-item questionnaire designed to measure levels of pain-catastrophizing. The scale comprises 13 items which yield an overall catastrophising score, which is a composite of magnification, rumination, and helplessness subscales. An overall score of greater than 24 is significant for pain catastrophising $(6,9,16)$

- Hospital Anxiety and Depression Scale (HADS). [Time Frame: 1 and 3 months, post-operative]

- HADS is a 14 item self-report questionnaire that was developed and found to be a reliable for detecting states of depression and anxiety in hospital patients, including those with cancer. A depression or anxiety score of greater than 10 is considered significant (17).

\section{Participant timeline $\{13\}$}

Time schedule of enrolment, interventions, assessments, and visits for participants are illustrated in Table 2.

Table 2: Time schedule for various points during study period. 


\begin{tabular}{|c|c|c|c|c|c|c|c|c|c|c|}
\hline \multirow[b]{3}{*}{ TIMEPOINT } & \multirow{3}{*}{\begin{tabular}{|l} 
Enrolment \\
$-t_{1}$
\end{tabular}} & \multicolumn{9}{|c|}{ STUDY PERIOD } \\
\hline & & \multirow{2}{*}{$\begin{array}{l}\text { Allocation } \\
0\end{array}$} & \multicolumn{6}{|c|}{ Post-allocation } & \multicolumn{2}{|c|}{$\begin{array}{l}\text { Primary \& } \\
\text { Secondary } \\
\text { outcomes }\end{array}$} \\
\hline & & & $\begin{array}{l}14 \text { days } \\
\text { pre-op }\end{array}$ & $\begin{array}{l}7 \text { days } \\
\text { pre- op }\end{array}$ & $\begin{array}{l}\text { Intra- } \\
\text { operative }\end{array}$ & $\begin{array}{l}24-48 \\
\text { hours } \\
\text { post-op }\end{array}$ & $\begin{array}{l}7 \text { days } \\
\text { post- op }\end{array}$ & \begin{tabular}{|l}
14 days \\
post- op
\end{tabular} & 30 days & 90 days \\
\hline \multicolumn{11}{|l|}{ ENROLMENT: } \\
\hline $\begin{array}{l}\text { Eligibility screen (Inclusion \& } \\
\text { exclusion criteria) }\end{array}$ & $\mathrm{X}$ & & & & & & & & & \\
\hline Informed consent & $\mathrm{X}$ & & & & & & & & & \\
\hline \multirow{2}{*}{$\begin{array}{l}\text { Baseline PCS } \\
\text { (>24: eligible to enrol) } \\
\text { Randomisation }\end{array}$} & $\mathrm{X}$ & & & & & & & & & \\
\hline & & $\mathrm{X}$ & & & & & & & & \\
\hline \multicolumn{11}{|l|}{ INTERVENTIONS } \\
\hline \multirow{2}{*}{$\begin{array}{l}\text { CBTsession group } \\
\text { PEM session group }\end{array}$} & & & $\mathrm{X}$ & $\mathrm{X}$ & & & $\mathrm{X}$ & $\mathrm{X}$ & & \\
\hline & & & $\mathrm{X}$ & $\mathrm{X}$ & & & $\mathrm{X}$ & $\mathrm{X}$ & & \\
\hline \multicolumn{11}{|l|}{ ASSESSMENTS: } \\
\hline \multirow{2}{*}{$\begin{array}{l}\text { Patient demographics and } \\
\text { characteristics }\end{array}$} & $\mathrm{X}$ & & & & & & & & & \\
\hline & & & & & $X$ & & & & & \\
\hline Intraoperative data & & $\mathrm{X}$ & & & & & & & $\mathrm{X}$ & $\mathrm{X}$ \\
\hline BPI & & & & & & $\mathrm{X}$ & & & & \\
\hline \multirow[t]{2}{*}{ QoR-15 } & & & & & & & & & & \\
\hline & & & & & & & & & $\Lambda$ & $x$ \\
\hline PCS & & & & & & & & & $\mathrm{X}$ & $\mathrm{X}$ \\
\hline
\end{tabular}

PCS- Pain Catastrophising Scale, CBT- Cognitive Behavioural Therapy, PEM- Pain Education \& Mindfulness, BPI: Brief Pain Inventory score, QoR-15- Quality of Recovery-15 score, HADS- Hospital anxiety and Depression Scale

\section{Sample size $\{14\}$}

The primary outcome is the difference in the BPI average pain severity score between the study groups, three months after surgery. A clinically important difference on the BPI is 2 raw score reduction on the 11-point Numerical Pain Rating Scale (NRS) (18-20)The standard deviation (SD) of BPI scores after breast surgery is in the order of 2.3 (21) on this scale. Taking a BPI score reduction of 2 as being clinically significant, then $n=21$ patients would be required each arm if Type I error $=0.05$ and Type II error is 0.2 (power $80 \%$ ). Taking into account of dropouts, we propose to enrol 24 eligible patients in each group ( $\mathrm{N}=48$ total). 


\section{Recruitment \{15\}}

Patients eligible for participation will be evaluated approximately two weeks before surgery and asked for informed consent, and then asked to complete the Pain Catastrophising Scale (PCS) questionnaire $(6,9,16)$ by a member of the research team. Patients with high pain catastrophising, defined as a score of $\geq 24$ on the PCS, and who satisfy all other eligibility criteria, will proceed for randomisation to either perioperative CBT or PEM groups. This cut-off is based on a previous study that showed a pre-treatment score of 24 or higher on the PCS best predicted follow-up chronic pain ratings and work status after multidisciplinary treatment (22) and has since been selected as an appropriate score cut-off in a perioperative CBT intervention in lumbar surgery patients (23). More recently, this cut-off was also suggested to obtain the highest sensitivity and specificity to predict unfavourable outcomes after spinal surgery (24).

\section{Assignment of interventions: allocation}

\section{Sequence generation $\{16 \mathrm{a}\}$}

Patients will be randomised after they are included in the study, having consented, and completed the PCS (and scoring scored $\geq 24$ ), and two preoperative appointments will be scheduled with the patients in the intervention and control groups. Patients will be randomised to either 'CBT-CP' or 'PEM groups by using an online computer-generated block randomisation.

\section{Concealment mechanism $\{16 \mathrm{~b}\}$}

Patient study number and group allocation will be typed onto separate pages and concealed in sequentially numbered sealed opaque envelopes.

\section{Implementation \{16c\}}

The randomisation process will be performed by an independent third party. The randomisation key/seed will also be held by an independent third party and investigators will not have access to this key/seed until the study has been completed, with the exception of the treating psychologist (DL) assigned to this trial as will have the responsibility in enrolling study participants and collecting baseline data. DL will allocate patients to either study intervention only after opening the sealed envelopes prospectively as participants are enrolled. DL will not have any additional involvement in data collection or analysis.

\section{Assignment of interventions: Blinding}

\section{Who will be blinded $\{17 \mathrm{a}\}$}

The treating psychologist will not be blinded as he is required to deliver both interventions associated with this trial. All other members of the research team involved in data collection and analysis will be blinded to the group allocation. In addition, patients will also be blinded because they will not be informed if they are receiving $\mathrm{CBT}$ or $\mathrm{PEM}$ other than that they are receiving one of two types of psychological interventions.

\section{Procedure for unblinding if needed $\{17 b\}$}


Un-blinding of trial participants to fellow research team members will occur only after creation of a final locked analysis dataset when the last patient has provided data at 3-month follow-up and after data has been statistically analysed by $(\mathrm{MBC}$ and $\mathrm{DB})$.=

\section{Data collection and management}

\section{Plans for assessment and collection of outcomes $\{18 \mathrm{a}\}$}

There will be three time points in which data collection will occur (preoperative, intraoperative, and postoperative). Data will be obtained from electronic and paper patient records. In addition, various questionnaires will be used to gather data to assess for the primary and secondary outcomes outlined for this trial (see section 12-outcomes).

\section{Plans to promote participant retention and complete follow-up \{18b\}}

During the screening process, potential participants will receive an extensive patient information leaflet about the study. The senior pain psychologist involved in this study will emphasise the importance of patient participation and the expectation to complete the study interventions and the various follow up questionnaires at the time of screening and enrollment to optimise retention and meaningful data collection.

\section{Data management $\{19\}$}

All data will be recorded on a study specific Case Report Form (CRF) that has the patient's unique study identifier code on each page. Study investigators will enter the data from CRF's into a designed study specific REDCap database that is password protected. Data will be entered primarily by one study investigator (AM) and verified by a different study investigator (CE) to minimize data entry errors (e.g., incorrect, or duplicate data). Both researchers will be blinded to group allocation.

The data collected and all the research-related documents (both hard copies and electronic copies) will be stored securely in a locked office in the Principal Investigator's office at the Mater Misericordiae University Hospital (MMUH). Only the principal investigator and the co-investigators can have access to these documents. The records will be kept for 5 years following study closure. All electronic files will be encrypted and accessed via password protected computers.

The electronic REDCap database also allows for specified ranges and automatic calculations to reduce entry errors. The study REDCap database will have automatic calculations for study questionnaires and specified ranges entered for each questionnaire response to ensure accurate data entry. Data will be cleaned by investigators upon completion of data collection to ensure good quality data.

\section{Confidentiality $\{27\}$}

All the data collected will remain anonymous and confidential. A unique subject number will be provided to each individual patient participating in the study. The front page of the CRF which will be labelled with both the randomisation number and patient information will be stored separately to the remainder of the CRF 
containing data about the patient to ensure data is re-identifiable. Only study investigators will have access to the data as CRF forms will be stored in a locked office that only study investigators have access to.

\section{Plans for collection, laboratory evaluation and storage of biological specimens for genetic or molecular analysis in this trial/future use $\{33\}$}

Not applicable, no samples will be collected.

\section{Statistical methods}

\section{Statistical methods for primary and secondary outcomes $\{20 \mathrm{a}\}$}

Outcome analyses will be conducted by researchers (MBC and DB) who will be blinded to treatment group allocation. Descriptive statistics will be calculated for all outcome measures at each time point, including for continuous variables: means, standard deviations, or medians with ranges of scores; and for categorical variables: frequencies and percentages.

Descriptive and inferential statistics will be obtained using the appropriate statistical methods required to address the study objectives. The primary analysis will compare the effect of the interventions on the primary outcomes; average pain severity at 3 months post-surgery. Outcome analyses will be conducted according to an intention to treat principle i.e. all randomised participants will be included in the main analysis and will be analysed as randomised, regardless of protocol adherence. Secondary analysis will involve the analysis of the secondary outcomes: QoR-15 at 24 hours post-surgery, PCS, HADS and average pain interference on the BPI short form at 3-months post-surgery. Linear mixed models on the outcome measures over time will be fitted to evaluate the effectiveness of both interventions, which intrinsically adjusts for baseline scores. Statistical significance will be assessed from a p-value $<0.05$ from the group by time interaction term. For all tests, 2sided $p$-values will be used, which will be reported to 4 decimal places with p-values less than 0.001 reported as $p<0.001$. In the case of a significant result, contrasts of the group effects at each assessment timepoint will be used to investigate the direction and pattern of effects. Irrespective of statistical significance, the mean changes and confidence intervals will be reported. An up-to-date version of SPSS will be used to conduct analyses.

\section{Interim analyses $\{21 \mathrm{~b}\}$}

No interim analyses will be conducted.

\section{Methods for additional analyses (e.g. subgroup analyses) \{20b\}}

A per-protocol analysis will exclude participants found to be ineligible after randomization and those who did not receive the intervention. Both intention to treat and per-protocol analyses will be reported and superiority will be determined only if demonstrated with the primary intention to treat analysis.

\section{Methods in analysis to handle protocol non-adherence and any statistical methods to handle missing data $\{20 \mathrm{c}\}$}


Careful attention will be paid to ensure that all participants are fully assessed at all time points to minimise missing data. We do not plan to use additional statistical methods such as multiple imputation as studies have demonstrated that linear mixed modelling is sufficient to control for missing data $(25,26)$

\section{Plans to give access to the full protocol, participant level-data and statistical code $\{31 \mathrm{c}\}$}

The collated data collected by the investigators will be retained for a maximum 5 years after analysis has been completed. We will deliver a completely de-identified data set an appropriate data upon reasonable request and in agreement with the principal investigator and data protection officer.

\section{Oversight and monitoring}

\section{Composition of the coordinating centre and trial steering committee $\{5 \mathrm{~d}\}$}

This is a single centre study. The trial steering committee will consist of the principal investigator (DB), trial coordinator (HK) and personnel responsible for data entry \& data management (AM, CE \& MBC). In addition, a research nurse employed by the institution, will also be a member of this committee (HK). This committee will meet monthly to evaluate the progress of the trial, address ongoing organizational and logistical issues and consider any adverse effects.

\section{Composition of the data monitoring committee, its role and reporting structure $\{21 \mathrm{a}\}$}

During the process of obtaining ethical approval for this single site study, a data protection impact assessment (DPIA) screening tool was completed. This was analyzed by the hospital's data protection officer (DPO) in MMUH. It was deemed that this study posed a low risk to the rights and freedoms of natural persons and therefore a formal DPIA was not needed. Recruitment of participants is expected to be completed within less than nine months or once the required number of participants needed is fulfilled. Due to the rapid expected inclusion of participants and the known minimal inherited risks associated with this trial, a data monitoring committee was not appointed.

\section{Adverse event reporting and harms $\{22\}$}

The study interventions associated with this trial does not involve any physical interventions and all patients will receive standard of care during their perioperative period. Therefore, it is not expected that any participants that volunteer to take part in this trial will suffer from any physical complications directly related from this study. However, some participants may find the study interventions (Cognitive Behavioural Therapy or Education and Mindfulness) distressing. This is not expected, but in the event if this occurs, it will be reported to the principal investigator. The participant will be removed from the trial if merited and further support will be provided.

\section{Frequency and plans for auditing trial conduct $\{23\}$}

A research nurse affiliated with $\mathrm{MMUH}$ anaesthesiology department but not involved in the trial by means patient recruitment, data collection, data entry and analysis will undertake an auditing process for trial 
conduct. This will occur every three months and would include: exploring the REDCap database for accuracy, proper data entry, duplicate data and adhering to data protection guidelines.

\section{Plans for communicating important protocol amendments to relevant parties (e.g. trial participants, ethical committees) $\{25\}$}

Approval for any study protocol amendments will be sought from the relevant IRB. Participant information leaflets will be updated accordingly and any changes to the published protocol will be reported in full in any future publications.

\section{Dissemination plans $\{31 \mathrm{a}\}$}

The results from this clinical trial will be fully disclosed by means of publication in an international peerreviewed journal and by oral/poster presentations at national and international scientific meetings. Both positive and negative findings will be disclosed.

\section{Discussion}

This paper describes the protocol of an RCT which will examine the effect of perioperative CBT on CPSP among breast cancer patients with high pain catastrophising characteristics. CPSP is a significant burden on patients that is also costly to healthcare systems. An intervention that has previously been shown to reduce CPSP such as the CBT, used in our trial may help to reduce this burden and associated costs to patients and healthcare systems globally. Our clinical trial aims to test the hypothesis that administrating a CBT intervention during the perioperative period is effective at reducing pain intensity at three months after breast cancer surgery, in high pain catastrophising patients. The authors will verify the aforementioned hypothesis by utilising quality controlled research methods through the use of a control group and sound statistical considerations and methods in relation to sample size, randomisation and data analysis. The use of CBT to address chronic post-surgical pain is a novel intervention in the field of surgical pain research that is highly relevant considering that up to $20-30 \%$ of breast cancer patients report CPSP at 6 months following surgery.

A recognised limitation for our study is, whilst the CBT-CP manualised module is typically delivered over eight to ten sessions, this study has modified it to fit within four appointments, at the expense of repeated instruction and with a particular focus on challenging pain-catastrophizing cognition.

Our study methods endeavour to prevent bias where possible via randomisation, concealment of allocation, specified procedures and efforts to reduce incomplete data, use of a statistically appropriate sample size and consistent thorough follow-up of study patients. The study results will contribute to current evidence in surgical pain treatment and management and inform current treatment practices and standards.

\section{Trial Status}

The trial is registered on ClinicalTrials.gov Identifier: NCT04924010. The current protocol is version 2 of $05 / 02 / 2021$. Participant recruitment began on $01 / 06 / 2021$ and full patient recruitment is estimated to be completed by July 2022 . Currently, $20 \%$ of patients have been recruited. 


\section{Abbreviations}

BPI

Brief Pain Inventory

CBT

Cognitive Behavioural Therapy

CBT-CT

Cognitive Behavioural Therapy for Chronic Pain

CPSP

Chronic Post-Surgical Pain

CRF

Case Report Form

DPIA

Data Protection Impact Assessment

DPO

Data Protection Officer

EMT

Educational and Mindfulness Therapy

HADS

Hospital Anxiety and Depression Scale

HSE

Health Service Executive

IRB

Institutional Review Board

MMUH

Mater Misericordiae University Hospital

NRS

Numerical Pain Rating Scale

PCS

Pain Catastrophising Scale

PEM

Pain Education and Mindfulness

PEC

Pectoralis

QoR-15

Quality of Recovery-15

SD

Standard Deviation

\section{Declarations}

Acknowledgements 
We would like to express our gratitude towards our current and future patients for their participation in this trial. In addition, we thank our nursing colleagues for their commitment and help in distributing the patient information leaflets and promoting this study. I

\section{Authors' contributions \{31b\}}

$D L, A M \& D J B$ were responsible for the study conception, design, proposal, and protocol development. AM is involved in the coordination for this trial. Both $A M \&$ CE are involved in the data collection process. CE \& MBC were involved in preparing the REDCap database and both will endeavor to ensure all data will be accurately entered. MBC will be involved in the statistical analysis for this trial. DL is the clinical psychologists involved in this study and is responsible for the design and implementing the study's interventions. With respect to the protocol, the manuscript was drafted by $A M, D L \& C E$ and was critically revised by MBC and DB. Both $A M$ and DL are Co-first authors and DB is the principal investigator. All authors read and approved the final manuscript.

\section{Funding $\{4\}$}

This trial is being funded internally from the Division of Anaesthesiology \& Perioperative Medicine, Mater University Hospital, Dublin. No external funding was received. Internal funders have no role in the design of this study, collection, management, analysis, or interpretation of data and writing of this manuscript.

\section{Availability of data and materials $\{29\}$}

The final trial dataset generated from this study will be made available from the corresponding and principal author on reasonable request.

\section{Ethics approval and consent to participate $\{24\}$}

Ethical approval has been granted from the Mater Misericordiae University Hospital Institutional Review Board (IRB) (Ref: $1 / 378 / 2217)$ on 12/05/2021. Consent to participate will be obtained from all participants prior to enrollment.

\section{Consent for publication $\{32\}$}

During the patient consent process, patients will be informed that the results of the study may be presented at national and/or international conferences or be published in an academic journal. Patients will be assured that no identifiable information will be presented or published. 


\section{Competing interests $\{28\}$}

The authors declare that they have no financial and non-financial competing interests.

\section{References}

1. Fletcher D, Stamer UM, Pogatzki-Zahn E, Zaslansky R, Tanase NV, Perruchoud C, et al. Chronic postsurgical pain in Europe: An observational study. Eur J Anaesthesiol. 2015;32(10):725-34. DOI:10.1097/EJA.0000000000000319.

2. Kehlet $\mathrm{H}$, Jensen TS, Woolf CJ. Persistent postsurgical pain: risk factors and prevention. Lancet. 2006;367(9522):1618-25. DOI:10.1016/S0140-6736(06)68700-X.

3. Spivey TL, Gutowski ED, Zinboonyahgoon N, King TA, Dominici L, Edwards RR, et al. Chronic Pain After Breast Surgery: A Prospective, Observational Study. Ann Surg Oncol. 2018;25(10):2917-24. DOI:10.1245/s10434-018-6644-x.

4. Weiser TG, Regenbogen SE, Thompson KD, Haynes AB, Lipsitz SR, Berry WR, et al. An estimation of the global volume of surgery: a modelling strategy based on available data. Lancet. 2008;372(9633):139-44. DOI:10.1016/S0140-6736(08)60878-8.

5. Vranceanu AM, Bachoura A, Weening A, Vrahas M, Smith RM, Ring D. Psychological factors predict disability and pain intensity after skeletal trauma. J Bone Joint Surg Am. 2014;96(3):e20. DOI:10.2106/JBJS.L.00479.

6. Leung L. Pain catastrophizing: an updated review. Indian J Psychol Med. 2012;34(3):204-17. DOI:10.4103/0253-7176.106012.

7. Wade JB, Riddle DL, Thacker LR. Is pain catastrophizing a stable trait or dynamic state in patients scheduled for knee arthroplasty? Clin J Pain. 2012;28(2):122-8. DOI:10.1097/AJP.0b013e318226c3e2.

8. Wideman TH, Sullivan MJ. Reducing catastrophic thinking associated with pain. Pain Manag. 2011;1(3):249-56. DOI:10.2217/pmt.11.14.

9. Osman A, Barrios FX, Gutierrez PM, Kopper BA, Merrifield T, Grittmann L. The Pain Catastrophizing Scale: further psychometric evaluation with adult samples. J Behav Med. 2000;23(4):351-65.

DOI:10.1023/a:1005548801037.

10. Villa G, Lanini I, Amass T, Bocciero V, Scire Calabrisotto C, Chelazzi C, et al. Effects of psychological interventions on anxiety and pain in patients undergoing major elective abdominal surgery: a systematic review. Perioper Med (Lond). 2020;9(1):38. DOI:10.1186/s13741-020-00169-x.

11. Giusti EM, Lacerenza M, Manzoni GM, Castelnuovo G. Psychological and psychosocial predictors of chronic postsurgical pain: a systematic review and meta-analysis. Pain. 2021;162(1):10-30. DOI:10.1097/j.pain.0000000000001999.

12. Connolly KS, Vanderploeg PS, Kerns RD, Grant C, Sellinger J, Godleski L. Nationwide Implementation and Outcomes of Cognitive Behavioral Therapy for Chronic Pain Over Clinical Video Teleconferencing. Journal of Technology in Behavioral Science. 2018;3(1):26-31. D0I:10.1007/s41347-017-0024-4. 
13. Murphy JL, McKellar JD, Raffa SD, Clark ME, Kerns RD, Karlin BE. Cognitive behavioral therapy for chronic pain among vetrans: therapist manual. Washington, DC: Department of Vetrans Affairs. [Available from: https://www.va.gov/painmanagement/docs/cbt-cp_therapist_manual.pdf.

14. Tan G, Jensen MP, Thornby JI, Shanti BF. Validation of the Brief Pain Inventory for chronic nonmalignant pain. J Pain. 2004;5(2):133-7. DOI:10.1016/j.jpain.2003.12.005.

15. Chazapis M, Walker EM, Rooms MA, Kamming D, Moonesinghe SR. Measuring quality of recovery-15 after day case surgery. Br J Anaesth. 2016;116(2):241-8. DOI:10.1093/bja/aev413.

16. sullivan MJ, Bishop SR, Pivik J. Pain Catastrophizing Scale: Development and validation. Psychol Assess. 1995;7(4):524-32. DOI:10.1037/1040-3590.7.4.524.

17. Annunziata MA, Muzzatti B, Bidoli E, Flaiban C, Bomben F, Piccinin M, et al. Hospital Anxiety and Depression Scale (HADS) accuracy in cancer patients. Support Care Cancer. 2020;28(8):3921-6. DOI:10.1007/s00520-019-05244-8.

18. Wong K, Zeng L, Zhang L, Bedard G, Wong E, Tsao M, et al. Minimal clinically important differences in the brief pain inventory in patients with bone metastases. Support Care Cancer. 2013;21(7):1893-9. DOI:10.1007/s00520-013-1731-9.

19. Marcus J, Lasch K, Wan Y, Yang M, Hsu C, Merante D. An Assessment of Clinically Important Differences on the Worst Pain Severity Item of the Modified Brief Pain Inventory in Patients with Diabetic Peripheral Neuropathic Pain. Pain Res Manag. 2018;2018:2140420. DOI:10.1155/2018/2140420.

20. Mease PJ, Spaeth M, Clauw DJ, Arnold LM, Bradley LA, Russell IJ, et al. Estimation of minimum clinically important difference for pain in fibromyalgia. Arthritis Care Res (Hoboken). 2011;63(6):821-6. DOI:10.1002/acr.20449.

21. Burckhardt CS, Jones KD. Effects of chronic widespread pain on the health status and quality of life of women after breast cancer surgery. Health Qual Life Outcomes. 2005;3:30. DOI:10.1186/1477-7525-3-30.

22. Scott W, Wideman TH, Sullivan MJ. Clinically meaningful scores on pain catastrophizing before and after multidisciplinary rehabilitation: a prospective study of individuals with subacute pain after whiplash injury. Clin J Pain. 2014;30(3):183-90. DOI:10.1097/AJP.0b013e31828eee6c.

23. Scarone P, Smeets A, van Kuijk SMJ, van Santbrink H, Peters M, Koetsier E. A randomized controlled TRlal of cognitive BEhavioral therapy for high Catastrophizing in patients undergoing lumbar fusion surgery: the TRIBECA study. BMC Musculoskelet Disord. 2020;21(1):810. DOI:10.1186/s12891-020-03826-w.

24. Tuna T, Boz S, Van Obbergh L, Lubansu A, Engelman E. Comparison of the Pain Sensitivity Questionnaire and the Pain Catastrophizing Scale in Predicting Postoperative Pain and Pain Chronicization After Spine Surgery. Clin Spine Surg. 2018;31(9):E432-E40. DOI:10.1097/BSD.0000000000000694.

25. Peters SA, Bots ML, den Ruijter HM, Palmer MK, Grobbee DE, Crouse JR 3. Multiple imputation of missing repeated outcome measurements did not add to linear mixed-effects models. J Clin Epidemiol. 2012;65(6):686-95. DOI:10.1016/j.jclinepi.2011.11.012. rd, et al.

26. Twisk J, de Boer M, de Vente W, Heymans M. Multiple imputation of missing values was not necessary before performing a longitudinal mixed-model analysis. J Clin Epidemiol. 2013;66(9):1022-8.

DOI:10.1016/j.jclinepi.2013.03.017. 


\section{Table 1}

Table 1: Summary of key components of cognitive behavioural therapy sessions delivered pre and post-surgery. 


\begin{tabular}{|c|c|c|}
\hline \multicolumn{3}{|l|}{ Pre-Surgery } \\
\hline Timing & $\operatorname{Aim}(\mathrm{s})$ & Content \\
\hline $\begin{array}{l}\text { Session } 1 \text { : } \\
\text { 1-2 weeks pre- } \\
\text { surgery } \\
60 \text { mins } \\
\text { Teleconference }\end{array}$ & $\begin{array}{l}\text { Establish rapport } \\
\text { Discussing treatment rationale } \\
\text { Pain Education } \\
\text { Relaxation methods }\end{array}$ & $\begin{array}{l}\text { Introductions } \\
\text { Gather personal history info } \\
\text { Discuss pain education re: relations } \\
\text { between cognition, emotions, behaviour, } \\
\text { and consequences } \\
\text { Introduce concept of fear avoidance } \\
\text { Build awareness on the potential impact of } \\
\text { stress and pain experience } \\
\text { Practice Relaxation Methods (PMR) } \\
\text { Homework: Monitoring cognitions, } \\
\text { emotions \& consequences; Identify } \\
\text { 'challenging' moments; Practice PMR }\end{array}$ \\
\hline $\begin{array}{l}\text { Session 2: } \\
1-3 \text { days pre- } \\
\text { surgery } \\
60 \text { mins } \\
\text { Teleconference }\end{array}$ & $\begin{array}{l}\text { Discuss surgery and associated fears / emotions/ } \\
\text { cognitions } \\
\text { Validate these fears and support person's } \\
\text { emotional state } \\
\text { Focus discussion on any catastrophizing thoughts } \\
\text { (e.g. "what if it goes wrong... " "something bad } \\
\text { might happen..." etc. }\end{array}$ & $\begin{array}{l}\text { Homework review } \\
\text { Relaxation practice } \\
\text { Defining 'catastrophizing' and 'state } \\
\text { anxiety' linking to any relevant homework } \\
\text { material } \\
\text { Explaining their importance in context of } \\
\text { surgery and recovery from } \\
\text { Practicing the identification of anxiety and } \\
\text { catastrophizing thoughts } \\
\text { Practicing cognitive restructuring } \\
\text { (challenging and replacing catastrophic } \\
\text { thoughts with more } \\
\text { adaptive/helpful/constructive 'facts') } \\
\text { End with PMR exercise } \\
\text { Homework: PMR; Continued mental } \\
\text { monitoring \& cog restructuring }\end{array}$ \\
\hline \multicolumn{3}{|l|}{ Post-Surgery } \\
\hline $\begin{array}{l}\text { Session 3: } \\
1-3 \text { days post- } \\
\text { surgery } \\
60 \text { mins } \\
\text { Teleconference }\end{array}$ & $\begin{array}{l}\text { Support participant post operatively } \\
\text { Validate their reported state of health/any } \\
\text { concerns } \\
\text { Maintain conversational focus on catastrophizing } \\
\text { tendencies, identifying them as they arise and } \\
\text { reinforcing the ability to challenge, restructure } \\
\text { etc. }\end{array}$ & $\begin{array}{l}\text { Homework review } \\
\text { Discussion of catastrophizing tendency } \\
\text { perioperatively and its links to } \\
\text { consequences } \\
\text { Discuss potential for fear avoidance and } \\
\text { link to consequences } \\
\text { Discuss goals and barriers } \\
\text { Framing conversation around adaptive } \\
\text { coping versus maladaptive coping } \\
\text { Acknowledge challenges } \\
\text { End with PMR exercise }\end{array}$ \\
\hline
\end{tabular}




\begin{tabular}{|l|l|l|} 
& & $\begin{array}{l}\text { Homework: Continued PMR practice; } \\
\text { Continued mental monitoring to detect and } \\
\text { challenge catastrophizing cognitions }\end{array}$ \\
\hline $\begin{array}{l}\text { Session 4: } \\
\text { surgery } \\
\text { 60mins } \\
\text { Teleconference }\end{array}$ & $\begin{array}{l}\text { Check-in } \\
\text { Maintaining progress } \\
\text { Promoting continued practice }\end{array}$ & $\begin{array}{l}\text { Homework review } \\
\text { Note and discuss progress } \\
\text { Validate scale of the experience } \\
\text { Contextualise content of thought processes } \\
\text { and encourage continued cognitive } \\
\text { restructuring in situations of } \\
\text { 'catastrophisation' } \\
\text { Reinforce continued PMR methods } \\
\text { Discuss medium term goals and barriers } \\
\text { Reviewing and summarising main learning } \\
\text { points from the last few weeks }\end{array}$ \\
\hline
\end{tabular}

\section{Figures}


Figure 1. Study flow chart

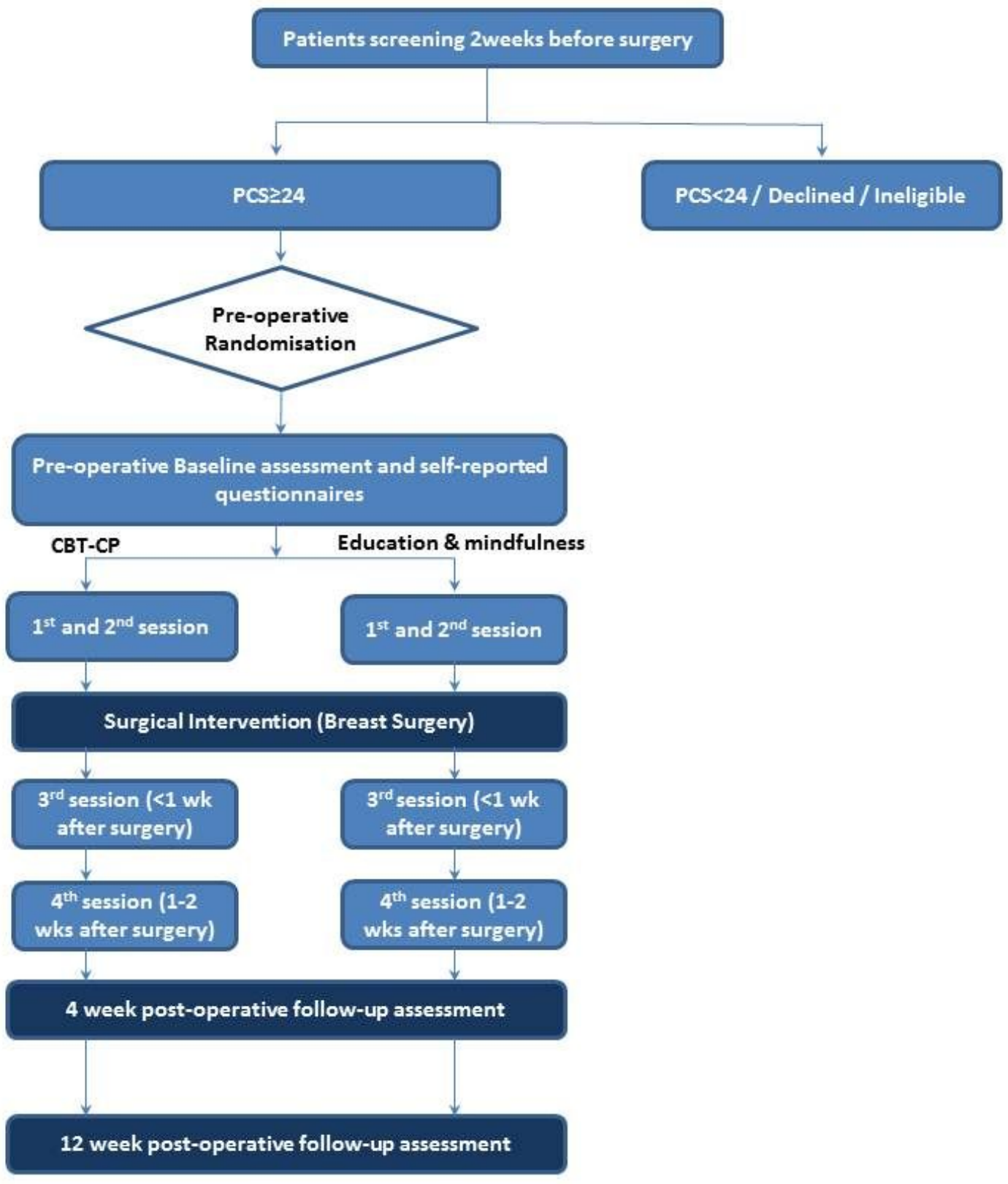

\section{Figure 1}

Study Flow Chart 